\title{
A Unified Model for Predicting the Open Hole Tensile and Compressive Strengths of Composite Laminates for Aerospace Applications
}

\author{
Paul Davidson* ${ }^{*}$ Evan J. Pineda ${ }^{\dagger}$, Christian Heinrich ${ }^{\ddagger}$, Anthony M. Waas ${ }^{\S}$ \\ University of Michigan, Ann Arbor, MI 48105, USA. Fellow AIAA
}

\begin{abstract}
The open hole tensile and compressive strengths are important design parameters in qualifying fiber reinforced laminates for a wide variety of structural applications in the aerospace industry. In this paper, we present a unified model that can be used for predicting both these strengths (tensile and compressive) using the same set of coupon level, material property data. As a prelude to the unified computational model that follows, simplified approaches, referred to as "zeroth order", "first order", etc. with increasing levels of fidelity are first presented. The results and methods presented are practical and validated against experimental data. They serve as an introductory step in establishing a virtual building block, bottom-up approach to designing future airframe structures with composite materials. The results are useful for aerospace design engineers, particularly those that deal with airframe design.
\end{abstract}

\section{Introduction}

Predicting the open hole compression strength (OHCS) and the open hole tensile strength (OHTS) of a laminated fiber reinforced composite is an important consideration in material qualification for aerospace structural design. Experimental results for fiber reinforced composite panels with holes and cut-outs show a significant drop in compressive strength as a function of hole size due to stress gradients generated by the hole and because of the large stresses that concentrate at the hole edge. The situation is similar for tensile loading.

In traditional multi-layered laminates, the failure in compression is generally dominated by the $0^{\circ}$ ply kink banding mechanism 1, while in tension, transverse cracks in the 90 degree layers, and matrix microcracks between fibers in off-axis layers are observed prior

\footnotetext{
*Graduate Student Research Assistant, Department of Mechanical Engineering. Student Member AIAA

†Senior Scientist, NASA Glenn Research Center, Member AIAA

${ }^{\ddagger}$ Senior Engineer, Sogeti Deutschland GmbH, Member AIAA

${ }^{\S}$ Felix Pawlowski Collegiate Professor, Department of Aerospace Engineering. Fellow AIAA
} 
to the onset of catastrophic failure, the latter resulting due to failure of the significant load-bearing zero plies, and sometimes also accompanied by delamination. Even though the problem of predicting compressive strength of laminated composite panels with a cutout is complex, it can be simplified by focussing attention on the failure of the $0^{\circ}$ lamina within the laminate. There are two main points that need to be taken into consideration; first the compressive strength of the $0^{\circ}$ lamina and second, the influence of the hole on the stress distribution around the hole. Prior studies, ${ }^{1,2,4,5}$ have examined the problem in detail and have developed both analytical and numerical methods based on careful experimental results to predict OHCS with varying degrees of sophistication in the modeling. In tension loading, in addition to transverse cracking in the 90 degree layers, matrix microcracking and fiber-matrix splitting in off-axis layers, and delaminations can all influence tensile strength. Hence, both damage and failure have to be taken into consideration in modeling for OHTS prediction.

At the zeroth order, we present a simple engineering approach using a combination of the Considere construction, ${ }^{7}$ and Lekhnitskii's ${ }^{8}$ formulation to approximate OHCS. For OHTS, we use a modified Lekhnitskii's analysis which uses a strain based failure index for predicting strength. At the first order, these models are refined by a combination of micromechanics and classical lamination theory, implemented using the finite element method to capture kink-band formation using a global-local approach, ${ }^{5}$ for accurate prediction of OHCS for a class of popular laminates. For OHTS, a similar modeling strategy with the smeared crack approach (SCA), described in Heinrich and Waas, ${ }^{13}$ can be used for a single fiber reinforced lamina to predict OHTS. For both cases, the modeling uses constitutive properties of the fiber and in-situ lamina shear-stress strain response. In the second order analysis, which has higher fidelity, we employ the thermodynamically-based Schapery work potential damage theory for capturing the in-plane damage in each lamina, and we also use the enhanced Schapery theory (EST), as described in Pineda et al, ${ }^{15}$ to model in-plane failure including post-peak softening using the crack band model. ${ }^{22}$ In this paper, results for OHCS are predicted using the zeroth, first and second order analysis methods, while results for OHTS are predicted using the zeroth order and second order analysis methods.

\section{Zeroth order analysis}

In multi-directional laminates, compression strength is generally dominated by the $0^{\circ}$ ply kink banding failure. Even though the problem of predicting compressive strength of laminated composite with a cut-out is complex, it can be simplified by focusing attention on the failure of $0^{\circ}$ laminae within the laminate. More specifically, the failure of $0^{\circ}$ laminae occurs at the location of maximum stress, which, in case of laminates with cut-outs like a hole, is at the hole edge. Hence, the simplest approach would involve; (a) knowing the compressive strength of $0^{\circ}$ lamina and, (b) knowing the value of stress at the hole edge. Therefore, the compressive strength of laminates with a hole is taken to be the applied stress which causes the maximum stress at the hole edge to reach the compressive strength of the $0^{\circ}$ lamina. This approach is implemented using a combination of the Considere construction, ${ }^{7}$

and Lekhnitskii's ${ }^{8}$ formulation. Considere construction is a geometric construction which utilizes the lamina shear stress-strain response to obtain the compressive strength of a $0^{\circ}$ lamina $\left(X_{c}^{0}\right)$ for a given fiber misalignment $\phi$, as shown in figure 2 . 


$$
X_{c}^{0}=\frac{\hat{\tau}_{12}}{\hat{\phi}+\hat{\gamma}_{12}}
$$

where, $\hat{\phi}$ is the fiber misalignment, $\hat{\tau}_{12}$ is the shear stress and $\hat{\gamma}_{12}$ shear strain. The compressive strength of a laminate, $X_{c}^{L}$, is obtained by,

$$
X_{c}^{L}=X_{c}^{0}\left(\frac{E_{11}^{L}}{E_{11}^{0}}\right)
$$

where, $E_{11}^{L}$ and $E_{11}^{0}$ are the elastic moduli of the laminate and the $0^{\circ}$ ply, respectively, in the direction of compressive load. Further, $X_{c}^{0}$ is the compressive strength of a $0^{\circ}$ lamina for a given fiber misalignment $\phi$.

The equation above provides the compressive strength of a laminate without any stressraisers (such as at cutout edges). In the presence of a hole, the maximum stresses occur at the hole edge due to non-uniform stress distribution. Using the Lekhnitskii ${ }^{8}$ solution, Filiou and Soutis, ${ }^{11}$ have provided an approximation to the stress distribution in an orthotropic infinite plate with a hole (shown in figure (3)). The equation below provides an accurate description of the axial stress, $\left(\sigma_{y y}(x, 0)\right)$ distribution due to a far field stress, $\sigma_{\infty}$,

$$
\begin{aligned}
& \frac{\sigma_{y y}(x, 0)}{\sigma_{\infty}} \approx 1+\frac{1}{2}\left(\frac{R}{x}\right)^{2}+\frac{3}{2}\left(\frac{R}{x}\right)^{4}-\frac{3}{2}\left(H_{A}-1\right)\left[5\left(\frac{R}{x}\right)^{6}-7\left(\frac{R}{x}\right)^{8}\right] \\
& H_{A}=\frac{(1+n)}{3} \\
& n=\sqrt{2\left(\sqrt{\frac{E_{y y}}{E_{x x}}}-\nu_{y x}\right)+\frac{E_{y y}}{G_{y x}}}
\end{aligned}
$$

Using the above equation, we can approximate the stress at the edge of the hole (for $x=R)$ as,

$$
\frac{\sigma_{y y}(R, 0)}{\sigma_{\infty}} \approx 3 H_{A}
$$

Assuming the material at the edge of the hole, where the stress is maximum, fails at the strength provided by equation 4 , above, ie; $\sigma_{y y}(R, 0) \approx X_{c}^{L}$ we can obtain the corresponding far field stress. This far field stress will be the critical strength of the laminate with a hole in compression, which corresponds to the zeroth order approximation.

$$
X_{c}^{L} \approx \frac{\sigma_{y y}(0, R)}{3 H_{A}}=\frac{X_{c}^{0}}{3 H_{A}}\left(\frac{E_{11}^{L}}{E_{11}^{0}}\right)
$$

Thus, this equation provides the OHCS of a laminate $\left(X_{c}^{L}\right.$, in terms of the pristine lamina compressive strength, $\left(X_{c}^{0}\right)$. The approach presented here can also be used to estimate the open hole compressive strength for other cutout shapes and for multiaxial planar loading, provided an expression that relates the maximum stress at the cutout edge in terms of the applied far-field loading is available.

To estimate OHTS, we utilize a similar approach except we assume that the laminate will fail when the strain in the $0^{\circ}$ ply reaches its ultimate tensile strain $\left(X_{\epsilon t}^{0}\right)$ obtained from a coupon $\left[0_{4}\right]$ tensile test. A uniform through-the-thickness strain is also assumed in this model. Equation 5 will now be modified to, 


$$
X_{t}^{L} \approx \frac{\sigma_{y y}(0, R)}{3 H_{A}}=\frac{E_{11}^{L} X_{\epsilon t}^{0}}{3 H_{A}}
$$

Equations 5-6 provide the zeroth order estimates for OHCS and OHTS. With the assumptions used for this simplified analysis, there is a significant drawback. The model does not account for material degradation due to in-plane damage and inter-laminar failure. Inplane damage and delamination is seen to be negligible in laminates with high percentage of $0^{\circ}$ plies, but that is not always the case with laminates with higher percentage of off-axis plies, such as quasi-isotropic laminates. This method should only be used as a starting point for the more refined methods that will be next described.

\section{First order analysis}

First order analysis is based on the same mechanism as the zeroth order approach, that the failure in laminates under compressive loading is due to kink banding. However, instead of analyzing the kink banding of a pristine $0^{\circ}$ lamina, and the hetrogeneous stress field due to a cut-out separately, the kink band formation in a $0^{\circ}$ lamina in a hetrogeneous stress field is analyzed. This is achieved through a micro-mechanical, finite element model of the $0^{\circ}$ lamina with a cut-out. In the micro-mechanics model, the fibers are explicitly modeled as homogeneous orthotropic continua and the matrix is modeled as an isotropic material with in-situ elastic-plastic properties. Fibers are misaligned by $1^{\circ}$ to account for average fiber misalignment angles commonly observed in carbon composite materials. ${ }^{3}$ This misalignment is the same as the one used in the zeroth level analysis. The micro-mechanics model, however, is a computationally expensive method, because of the difference in length scale of fiber and lamina. To model a complete lamina using micro-mechanics will require thousands of fiber-matrix layers, which is computationally prohibitive. To overcome the issue of computational cost the model is reduced by analysing a micro-region around the hole. Boundary conditions to be applied on the micro-region are obtained from a global homogeneous model. This method is called the global-local approach as defined in the work by Ahn and Waas $^{5}$ and Davidson and Waas. ${ }^{6}$ In some reports, this method has also been referred to as the embedded cell method (see, for example, Llorca et a ${ }^{19}$ ).

The local-global approach implemented here is a three step procedure (each subsequent step relies on the results of the prior step) using a macro-mechanical model and this is as depicted in figure(4). Each step is an individual simulation and only the boundary information is passed from one step to the other. The macro-model, outside the micromechanics sub-region, is a 2D half symmetric model with homogenized transversely isotropic lamina properties of the $0^{\circ}$ lamina. Step 1, is a linear elastic step where a known far-field displacement is applied in the axial $(y)$ direction and the displacements $\left(\Delta_{x, y}\right)$ at nodes corresponding to the micro-region boundary $(A-B-C-D)$ are obtained. These displacements $\Delta_{x, y}$ are then applied on the isolated local micro-mechanics model boundary and another linear elastic analysis is performed in step 2, henceforth called "Local Linear Model". In step 3, the reaction forces, $\left(R_{x, y}\right)$, obtained in step 2 are applied on boundaries $(A-D) \&(B-C)$ along with $\Delta_{x, y}$ on $(A-B) \&(C-D)$ and a non-linear Riks response analysis is conducted ("Local Non-linear Model") on the isolated local micro-mechanics model. Only step 3 is an incremental analysis while steps 1 and 2 are linear and hence done only once. Step 2 is needed

to obtain the consistent reaction forces on parts of the boundary of the micro-region, since 
step 3 uses mixed boundary conditions on the micro-region boundary.

Typically, as seen in past studies, there are no boundary conditions imposed on the free edge of a local model. This however, can lead to incorrect results because the local model, which is hetrogeneous, will deform in a manner different from the global model. In figure 5, the un-deformed and deformed profiles of global and local model are shown. Pinching of the corners of the micro-region is observed as compared to the global model, during deformation, which will lead to artificial stress concentration developing at the corners. These stress concentrations will cause premature kink formation at the corners. Hence, application of reaction forces at the free boundaries is important to ensure that the local model follows the global deformation profile, in the elastic regime. By applying reaction forces instead of displacements, the left and right boundaries are kept free of constraints, thereby allowing for the kink band to form without displacements being constrained on the boundaries.

The axial reaction force $R_{y}$ obtained from steps $1 \& 2$ provide the linear relation between the local and global model as a simple ratio $\hat{R}=\left(R_{y}^{\text {global }} / R_{y}^{\text {local }}\right)$. Now, from step 3 , we get the micro-kink band initiation load $\left(R_{k}^{\text {local }}\right)$ which corresponds to the global compressive strength of the zero lamina due to kink banding, $X_{N}^{0}$ as ;

$$
X_{N}^{0}=\left(R_{k}^{\text {global }}\right) / A=\left(\hat{R} R_{k}^{\text {local }}\right) / A
$$

In the above expression, $A$, is the load bearing cross-sectional area of the specimen in the far field. The size of the micro (local) region is an unknown of the global-local method. A converged solution is obtained when the peak compressive strength of the first order analysis shows no difference with respect to the size of the micro-region. The size of the region was scaled based on the ratio of width (length A-B) to the height (length B-C) of the micro-region.

Typical response of the micro-region is shown in figure (6) and corresponding snapshots at different stages of loading leading to kink banding failure are provided. Figure (8) shows the scaling study to determine the converged micro-region size and the corresponding compressive strength of the $0^{\circ}$ ply. The results are normalized by the compressive strength of the $0^{\circ}$ ply with $\phi=1^{\circ}$ obtained from the Considere construction ie; the un-notched zero ply strength. Using the converged value of compressive strength from the $2 \mathrm{D}$ global local $X_{N}^{0}$ analysis, the laminate compressive strength can now be obtained by simple scaling,

$$
X_{c}^{L}=X_{N}^{0}\left(\frac{E_{11}^{L}}{E_{11}^{0}}\right)
$$

As with zeroth order, this first order approach provides compressive strength prediction for laminates that show kink band formation in the $0^{\circ}$ lamina. Hence, this method of predicting compressive strength is predicated on zero ply kink banding being the dominant failure mechanism. However, this may not provide satisfactory predictions in cases where off-axis laminae dominate, ie; in cases where the percentage of $0^{\circ}$ laminae is small and where there is significant non-linear behaviour before failure, as in pure angle-ply laminates or laminates with a large percentage of angle plies. 


\section{Second order analysis}

Zeroth and first order analysis methods do not account for stiffness degradation of off-axis laminae. The difficulty in analytically modeling off-axis plies is because significant damage accumulates in these plies during loading. A second order modeling method should capture both damage and failure. This is achieved by modeling damage using Schapery theory and failure using the crack-band model, ${ }^{22}$ which is referred to as Enhanced Schapery Theory (EST).

The EST formulation developed by Pineda et $\mathrm{al}^{15}$ is used, which assumes that three major intralaminar mechanisms are responsible for all observed non-linearities in the stress-strain curve of a composite lamina: matrix microdamage, matrix macroscopic cracking (modes I and II), and axial fiber failure (mode I). Each of these mechanisms can be accommodated by partitioning the total dissipated energy density, $W_{S}$, into portions associated with each mechanism. It is assumed that the evolution of these failure mechanisms (i.e., macroscopic cracking and fiber breakage) yields an immediate reduction in the load-carrying capability of a local subvolume where the mechanism is active.

EST in combination with Cohesive Zone methods, such as the discrete cohesive zone method (DCZM),${ }^{20}$ can be used to account for both in-plane and inter-ply damage. However, failure due to delamination is not considered in the second order analysis, since the material system studied here did not exhibit significant delamination.

\section{A. EST Evolution Equations for a Fiber-Reinforced Lamina}

EST is a multiple-internal state variable (ISV) formulation, which utilizes separate ISVs for modeling the effects of damage and failure. Four ISVs are used; $S$, for damage, $S_{I}^{f}$, for fiber failure, $S_{I}^{m}$, for transverse matrix failure, and $S_{I I}^{m}$ for matrix shear failure. To arrive at the evolution equations for the ISVs, the elastic strain energy density must be defined for a material, which may contain cohesive cracks. Therefore, the elastic strain energy $W_{E}$ is comprised of a contribution from the continuum $W$ and any possible cohesive cracks $W_{M}^{j}$. The plane stress, elastic strain energy density in the continuum is defined as,

$$
W=\frac{1}{2}\left(E_{11} \epsilon_{11}^{2}+E_{22}(S) \epsilon_{22}^{2}+G_{12}(S) \gamma_{12}^{2}\right)+Q_{12} \epsilon_{11} \epsilon_{22}
$$

where stress in the laminae are related to strain assuming plane stress conditions.

$$
\begin{aligned}
& \sigma_{11}=Q_{11} \epsilon_{11}+Q_{12} \epsilon_{22} \\
& \sigma_{22}=Q_{12} \epsilon_{11}+Q_{22} \epsilon_{22} \\
& \tau_{12}=Q_{66} \gamma_{12}
\end{aligned}
$$

where $\gamma_{12}$ is the engineering shear strain and

$$
\begin{aligned}
Q_{11} & =\frac{E_{11}}{1-\nu_{12} \nu_{21}} \\
Q_{22} & =\frac{E_{22}}{1-\nu_{12} \nu_{21}} \\
Q_{12} & =\nu_{12} Q_{22} \\
Q_{66} & =G_{12} \\
\nu_{21} & =\frac{\nu_{12} E_{22}}{E_{11}}
\end{aligned}
$$

6 of 26 
where $E_{11}$ is the axial elastic modulus, $E_{22}$ is the transverse elastic modulus, $\nu_{12}$ is the Poisson's ratio, $\nu_{21}$ is the transverse Poisson's ratio, and $G_{12}$ is the elastic shear modulus. After assuming that the quantity $\nu_{12} \nu_{21}<<1$, Equations (11) simplify,

$$
\begin{aligned}
& Q_{11}=E_{11} \\
& Q_{22}=E_{22} \\
& Q_{12}=\nu_{12} Q_{22} \\
& Q_{66}=G_{12}
\end{aligned}
$$

Note that only the transverse and shear moduli $\left(E_{22}\right.$ and $\left.G_{12}\right)$ are functions of $S$ since matrix microdamage only accrues in the matrix of the laminae. The Poisson's ratio is assumed to evolve such that the quantity $Q_{12}=E_{22} \nu_{12}$ remains constant; however, this restriction can be relaxed if deemed necessary. The degraded moduli are related to the virgin moduli $\left(E_{220}\right.$ and $\left.G_{120}\right)$ and the ISV through a set of microdamage functions $\left(e_{s}(S)\right.$ and $\left.g_{s}(S)\right)$.

$$
\begin{aligned}
& E_{22}=E_{220} e_{s}(S) \\
& G_{12}=G_{120} g_{s}(S)
\end{aligned}
$$

Degrading $E_{22}$ and $G_{12}$ exclusively is consistent with the intralaminar damage typically observed in PMC laminates.

The elastic strain energy density of the cohesive cracks is defined as the recoverable energy per unit crack surface area smeared over the entire element.

$$
\begin{gathered}
W_{I}^{f}=\frac{t_{I}^{f} \delta_{I}^{f}}{2 l_{e}^{\left(\theta+90^{\circ}\right)}} \\
W_{I}^{m}=\frac{t_{I}^{m} \delta_{I}^{m}}{2 l_{e}^{(\theta)}} \\
W_{I I}^{m}=\frac{t_{I I}^{m} \delta_{I I}^{m}}{2 l_{e}^{(\theta)}}
\end{gathered}
$$

where, $\delta_{I}^{f}$ is the fiber direction effective crack opening displacement, $\delta_{I}^{m}$ is the matrix transverse direction effective crack opening displacement, and $\delta_{I I}^{m}$, is the matrix shear direction effective crack opening displacement. $l_{e}^{\left(\theta+90^{\circ}\right)}$ is the length of a line running perpendicular to fiber direction in the element that intersects two edges of the element and the integration point, and $l_{e}^{(\theta)}$ is the length of a line that is parallel to the fiber direction in the element that intersects two edges of the element and the integration point. The cohesive cracks are not active until some initiation criterion is met. Prior to that, the crack tip opening displacements $\delta_{M}^{j}$ are zero, and the do not contribute to the elastic strain energy density. The tractions in Equations (15)-(17) can be related to the secant stiffness' using triangular traction-separation laws, $k_{M}^{j}$ shown in figure 7 .

$$
\begin{gathered}
t_{I}^{f}=k_{I}^{f} \delta_{I}^{f} \\
t_{I}^{m}=k_{I}^{m} \delta_{I}^{m} \\
t_{I I}^{m}=k_{I I}^{m} \delta_{I I}^{m}
\end{gathered}
$$


Hence, the total elastic strain energy density in the continuum is given by

$$
\begin{aligned}
W_{E}= & \frac{1}{2}\left(E_{11} \epsilon_{11}^{2}+E_{22}(S) \epsilon_{22}^{2}+G_{12}(S) \gamma_{12}^{2}\right)+Q_{12} \epsilon_{11} \epsilon_{22} \\
& +\frac{k_{I}^{f}\left(S_{I}^{f}\right)}{2 l_{e}^{\left(\theta+90^{\circ}\right)}} \delta_{I}^{f^{2}}+\frac{k_{I}^{m}\left(S_{I}^{m}\right)}{2 l_{e}^{(\theta)}} \delta_{I}^{m 2}+\frac{k_{I I}^{m}\left(S_{I I}^{m}\right)}{2 l_{e}^{(\theta)}} \delta_{I I}^{m 2}
\end{aligned}
$$

Invoking the stationarity of potential with respect to each ISV, gives the ISV evolution equations.

$$
\begin{gathered}
\frac{1}{2}\left(\epsilon_{22}^{2} E_{220} \frac{d e_{s}}{d S_{r}}+\gamma_{12}^{2} G_{120} \frac{d g_{s}}{d S_{r}}\right)=-3 S_{r}^{2} \\
\frac{1}{2 l_{e}^{\left(\theta+90^{\circ}\right)}} \frac{d k_{I}^{f}}{d S_{I}^{f}} \delta_{I}^{f^{2}}=-1 \\
\frac{1}{2 l_{e}^{(\theta)}} \frac{d k_{I}^{m}}{d S_{I}^{m}} \delta_{I}^{m 2}=-1 \\
\frac{1}{2 l_{e}^{(\theta)}} \frac{d k_{I I}^{m}}{d S_{I I}^{m}} \delta_{I I}^{m 2}=-1
\end{gathered}
$$

The use of a reduced ISV $S_{r}=S^{\frac{1}{3}}$ has been employed in Equation (22). Sicking ${ }^{21}$ has shown that the use of this reduced ISV yields polynomial forms of the microdamage functions in Equations (13) and (14). Thus, Equation (22) becomes a polynomial equation that can be readily solved for $S_{r}$ for a given strain state $\left(\epsilon_{22}, \gamma_{12}\right)$. No mixed-mode law is incorporated in this work; thus, mode I and mode II failures are uncoupled. Future incrementation of EST will implement a mixed-mode fracture law to introduce this coupling. However, since the mode I and mode II displacements are uncoupled here, $S_{I}^{f}, S_{I}^{m}$, and $S_{I I}^{m}$ can be determined from Equations (23) - (25), a priori, in terms of the applied strain using the traction separation laws and kinematics.

Using the chain rule and the fact that

$$
\begin{gathered}
\frac{d S_{I}^{f}}{d \delta_{I}^{f}}=\frac{t_{I C}^{f}}{2 l_{e}^{\left(\theta+90^{\circ}\right)}} \\
\frac{d S_{I}^{m}}{d \delta_{I}^{m}}=\frac{t_{I C}^{m}}{2 l_{e}^{(\theta)}} \\
\frac{d S_{I I}^{m}}{d \delta_{I I}^{m}}=\frac{t_{I I C}^{m}}{2 l_{e}^{(\theta)}}
\end{gathered}
$$

by traction separation laws, ${ }^{15}$ the cohesive secant stiffnesses are determined. The cohesive strengths of the material are $t_{I C}^{f}$ (mode I fiber strength), $t_{I C}^{m}$ (mode I matrix strength), and $t_{I I C}^{m}$ (mode II matrix strength), and

$$
\begin{aligned}
k_{I}^{f} & =-\int \frac{t_{I C}^{f}}{\delta_{I}^{f^{2}}} d \delta_{I}^{f} \\
k_{I}^{m} & =-\int \frac{t_{I C}^{m}}{\delta_{I}^{m^{2}}} d \delta_{I}^{m}
\end{aligned}
$$




$$
k_{I I}^{m}=-\int \frac{t_{I I C}^{m}}{\delta_{I I}^{m 2}} d \delta_{I I}^{m}
$$

Evaluating the integrals in Equations (29)-(31), while enforcing $k_{M}^{j}=0$ when $\delta_{M}^{j}=\frac{2 G_{M C}^{j}}{t_{M C}^{j}}$ results in expressions for $k_{M}^{j}$ in terms of $\delta_{M}^{j}$.

$$
\begin{gathered}
k_{I}^{f}=t_{I C}^{f}\left(\frac{1}{\delta_{I}^{f}}-\frac{t_{I C}^{f}}{2 G_{I C}^{f}}\right) \\
k_{I}^{m}=t_{I C}^{m}\left(\frac{1}{\delta_{I}^{m}}-\frac{t_{I C}^{m}}{2 G_{I C}^{m}}\right) \\
k_{I I}^{m}=t_{I I C}^{m}\left(\frac{1}{\delta_{I I}^{m}}-\frac{t_{I I C}^{m}}{2 G_{I I C}^{m}}\right)
\end{gathered}
$$

where $G_{I C}^{f}$ is the mode I fracture toughness of the fiber, $G_{I C}^{m}$ is the mode I fracture toughness of the matrix, and $G_{I I C}^{m}$ is the mode II fracture toughness of the matrix. The thermodynamically consistent stiffnesses derived in Equations (32)-(34) can also be derived directly from the traction-separation laws using geometry.

Finally, it is assumed that following failure initiation, the strains are related to the crack tip opening displacements by

$$
\begin{gathered}
l_{e}^{\left(\theta+90^{\circ}\right)} \epsilon_{11}=l_{e}^{\left(\theta+90^{\circ}\right)} \epsilon_{11}^{C}+\delta_{I}^{f} \\
l_{e}^{(\theta)} \epsilon_{22}=l_{e}^{(\theta)} \epsilon_{22}^{C}+\delta_{I}^{m} \\
l_{e}^{(\theta)} \gamma_{12}=l_{e}^{(\theta)} \gamma_{12}^{C}+2 \delta_{I I}^{m}
\end{gathered}
$$

where $\epsilon_{11}^{C}, \epsilon_{22}^{C}$, and $\gamma_{12}^{C}$ are the strains when, the Hashin-Rotem matrix failure criterion, involving contributions from both the transverse $\left(\epsilon_{22}\right)$ and shear $\left(\gamma_{12}\right)$ strains, is satisfied.

$$
\begin{aligned}
& \left(\frac{\epsilon_{22}}{Y_{T}}\right)^{2}+\left(\frac{\gamma_{12}}{Z}\right)^{2}=1 \quad \epsilon_{22} \geq 0 \\
& \left(\frac{\epsilon_{22}}{Y_{C}}\right)^{2}+\left(\frac{\gamma_{12}}{Z}\right)^{2}=1 \quad \epsilon_{22}<0
\end{aligned}
$$

$Y_{T}$ is the transverse lamina failure strain in tension, $Y_{C}$ is the transverse failure lamina strain in compression, and $Z$ is the shear failure strain. The fiber failure criterion only involves the axial strain $\epsilon_{11}$.

$$
\left(\frac{\epsilon_{11}}{X_{T}}\right)^{2}=1 \quad \epsilon_{11} \geq 0
$$

where $X_{T}$ is the maximum allowable axial strain of the lamina. The Hashin-Rotem initiation criterion is utilized here for its tractability and computational efficiency, but virtually any initiation criterion could be used to govern the onset of matrix failure due to macroscopic cracking or axial failure due to fiber breakage.

Equations (35)-(37) imply that the strain in the continuum remains at the values obtained when failure initiates, and that any incremental change in the global strain after failure 
initiation is used wholly to advance the crack tip opening displacement. To account for changes in the continuum strain after failure initiates, it can be assumed that the stress state in the cracked body is homogenous and the tractions on the crack tip faces are equal to the stresses in the continuum. Then, the strains in Equation (21) can be formulated in terms of the cohesive secant stiffnesses and the crack tip opening displacement. However, it is assumed that the evolution of strain in the continuum is negligible once cohesive cracks form. Equations (35)-(37) can be utilized in Equations (32)-(34) to obtain $k_{M}^{j}$ as functions of the global strain at an integration point.

$$
\begin{gathered}
k_{I}^{f}=t_{I C}^{f}\left[\frac{1}{l_{e}^{\left(\theta+90^{\circ}\right)}\left(\epsilon_{11}-\epsilon_{11}^{C}\right)}-\frac{t_{I C}^{f}}{2 G_{I C}^{f}}\right] \\
k_{I}^{m}=t_{I C}^{m}\left[\frac{1}{l_{e}^{(\theta)}\left(\epsilon_{22}-\epsilon_{22}^{C}\right)}-\frac{t_{I C}^{m}}{2 G_{I C}^{m}}\right] \\
k_{I I}^{m}=t_{I I C}^{m}\left[\frac{2}{l_{e}^{(\theta)}\left(\gamma_{12}-\gamma_{12}^{C}\right)}-\frac{t_{I I C}^{m}}{2 G_{I I C}^{m}}\right]
\end{gathered}
$$

Once failure initiates, the effects of failure supersede the effects of microdamage and evolution of $S$ ceases. The cohesive stiffness in a cracked element is calculated using Equations (40)-(42) for a given strain state; then, Equations (18)-(20) and (35)-(37) are used to calculate the tractions on the crack tip faces and the crack tip opening displacement. It is assumed that the stress state in the integration point subvolume of the element is homogenous, and the tractions on the crack tip faces are equal to the stresses in the element. Lastly, the axial, transverse, and shear moduli of the element can be calculated: ${ }^{22}$

$$
\begin{aligned}
& E_{11}=\left\{\frac{1}{E_{110}}-\frac{\epsilon_{11}-\epsilon_{11}^{C}}{t_{I C}^{f}\left[1+\frac{l_{e}^{\left(\theta+90^{\circ}\right)} t_{I C}^{f}}{2 G_{I C}^{f}}\left(\epsilon_{11}-\epsilon_{11}^{C}\right)\right]}\right\}^{-1} \\
& E_{22}=\left\{\frac{1}{E_{22}^{*}}-\frac{\epsilon_{22}-\epsilon_{22}^{C}}{t_{I C}^{m}\left[1+\frac{l_{e}^{(\theta)} t_{I C}^{m}}{2 G_{I C}^{m}}\left(\epsilon_{22}-\epsilon_{22}^{C}\right)\right]}\right\}^{-1} \\
& G_{12}=\left\{\frac{1}{G_{12}^{*}}-\frac{\gamma_{12}-\gamma_{12}^{C}}{2 t_{I I C}^{m}\left[1+\frac{l_{e}^{(\theta)} t_{I I C}^{m}}{4 G_{I I C}^{m}}\left(\gamma_{12}-\gamma_{12}^{C}\right)\right]}\right\}^{-1}
\end{aligned}
$$

where $E_{22}^{*}$ and $G_{12}^{*}$ are the degraded transverse and shear moduli, due to microdamage, when Equation (38) is satisfied. 
It should be noted that EST also accounts for kink band formation under axial compression. ${ }^{23-25}$ As the lamina is loaded, the fibers in the composite rotate by some angle $\phi$, and attain the new direction $\mathbf{e}_{\mathbf{f}}$, given by the deformation gradient $\mathbf{F}$ in the model.

$$
\mathbf{e}_{\mathbf{f}}=\frac{1}{\lambda} \mathbf{F} \cdot \mathbf{e}_{\mathbf{f 0}}
$$

where $\mathbf{e}_{\mathbf{f o}}$ is the fiber orientation in the undeformed configuration and $\lambda$ is the stretch ratio.

$$
\lambda=\sqrt{\mathbf{e}_{\mathbf{f 0}} \mathbf{F F}^{\mathbf{T}} \mathbf{e}_{\mathbf{f 0}}}
$$

To model the kink band mechanism, all calculations are then executed in the instantaneous fiber frame; therefore, fiber rotation can induce large shear strains, $\gamma_{12}$. As the shear strain increases, more damage is induced leading to a reduction in the local tangent shear modulus. The increase in local shear compliance allows for further increase in the shear strain. Under increasing axial compression, this mechanism leads to a runaway (negative feedback) instability, and a kink band eventually precipitates.

In summary, Equations (38) and (39) mark the transition from evolving microdamage to failure to macroscopic cracking. Prior to failure initiation, Equation (22) is used to calculate the microdamage reduced ISV $S_{r}$, and the failure ISVs $S_{I}^{f}, S_{I}^{m}$, and $S_{I I}^{m}$ remain zero. Equations (13) and (14) are used to calculate the degraded transverse and shear moduli. Subsequent to matrix failure initiation, microdamage growth is precluded, and $S_{r}$ remains at $S_{r}^{*}$, the value of $S_{r}$ when Equation (38) was satisfied. The degeneration of the transverse and shear moduli, resulting from matrix transverse and shear cracking, is calculated using Equations (44) and (45). Finally if Equation (39) is satisfied, the axial modulus is calculated using Equations (43) as fiber breakage evolves in the element. Once the material moduli have been calculated using the appropriate evolution equations, the stresses can be updated accordingly using Equations (12).

\section{Material damage and failure characterization}

General procedures for laminates with a cut-outs, like a hole, are shown in figure(9). There are two stages to the analysis, first is the measurement and modeling to obtain input parameters from coupon level tests and micromechanics predictions. These are then used in the second stage, which is the prediction of OHCS, OHTS and any other progressive failure scenario. There are four categories of inputs(figure 9), geometry, elastic material properties, damage properties, and failure properties. Some of the parameters are measured while others are derived based on the measured values. Different input details are required in each category ranging from constitutive fiber and matrix properties in the micro level to the laminate properties at the coupon level as shown in figure 10.

Due to propriety nature of the composite material used, no information about the material will be provided. However, a complete set of EST input and respective test requirements are listed in table 1 , and are described in subsequent sections.

\section{A. Measuring lamina in-situ shear response}

In all the methods described, the common and critical data required is the in-situ elasticdamage response of the matrix. Characterization of the in-situ lamina shear response is done 
using the procedure described by $\mathrm{Ng}$ et al. ${ }^{9}$ Tensile tests of dog-bone shaped specimens of $\pm 45^{\circ}{ }_{s}$ were conducted to obtain the in-plane lamina shear response, and the tests followed the guidelines of ASTM D3518 and D3039 for strain gage requirements and gage section dimensions. Dogbone-shaped specimens were used rather than standard coupon-shaped specimens because previous work showed a tendency of specimen failure within the grips for straight-sided coupons and within the gage section for dog-bone coupons.

A speckle pattern was also applied on one side of the specimens to capture full field strain data using an optical camera. Subsequently, the speckle images were analyzed using the ARAMIS digital image correlation (DIC) software. Strain gage data was compared to, and agreed with, the strains obtained through DIC. However, strain gages did not cover the full range of strains seen in the experiments and failed prior to sample ultimate failure. Hence, DIC measurements were used for calculating full field strain data and used for the analysis.

Using the shear response of the lamina, the in-situ shear response of the damaging matrix, which is shown in figure (11a) was extracted as described in. ${ }^{9}$ From this, the in-situ matrix equivalent stress vs equivalent strain response can be calculated $\left(\mathrm{Ng}\right.$ et $\mathrm{al}^{9}$ ) and is shown in figure (11b).

\section{B. Damage parameters}

Items 1-3 in table 1 refer to the Schapery damage evolution curves described by the polynomial forms of $e_{s}$ and $g_{s}$ as

$$
\begin{aligned}
& e_{s}\left(S_{r}\right)=e_{s 0}+e_{s 1} S_{r}+e_{s 2} S_{r}^{2}+e_{s 3} S_{r}^{3}+e_{s 4} S_{r}^{4}+e_{s 5} S_{r}^{5} \\
& g_{s}\left(S_{r}\right)=g_{s 0}+g_{s 1} S_{r}+g_{s 2} S_{r}^{2}+g_{s 3} S_{r}^{3}+g_{s 4} S_{r}^{4}+g_{s 5} S_{r}^{5}
\end{aligned}
$$

The shear microdamage function $g_{s}$ was obtained from the shear stress-shear strain $\left(\tau_{12}-\gamma_{12}\right)$ response of $\left[45^{\circ} /-45^{\circ}\right]$ angle-ply coupon tests as recommended by Sicking. ${ }^{21}$ The transverse tensile micro-damage function $e_{s}$ requires testing of $\left[30^{\circ} /-30^{\circ}\right]$ angle-ply coupon tests, also recommended by Sicking. ${ }^{21}$ However, $e_{s}$ can also be obtained from the in-situ matrix equivalent stress-equivalent strain response. Using the non-linear in-situ matrix properties in a cubic close-packed repeating unit cell, and applying transverse loading, the lamina transverse response curve can be obtained. The transverse non-linear response curve is then used to calculate $e_{s}$ following Sicking's procedure. ${ }^{21}$ The two curves are shown in figure 12a $\& 12 \mathrm{~b}$.

\section{Intralaminar failure strain}

Intralaminar failure strains are measured using DIC analysis of tests listed in items 4,6 and 8 of table 1. Compressive failure strain in the fiber direction can be obtained from [0] compression tests, which require thicker coupons such that kink band formation can be captured using DIC. Alternatively, compressive failure strain can instead be found using a micro-mechanics finite element model $(\mathrm{FEM})^{17}$ or analytical model ${ }^{16}$ of the composite. The transverse compression response (item 7 ) is generally taken to be the same as in tension, but the failure strengths are different and the tensile failure strength (or strain) is measured from a coupon test. 


\section{Intralaminar failure toughness}

Fiber direction tension failure toughness can be measured from Compact Tension Specimen $(\mathrm{CTS})^{18}$ or Single Edge Notch Tension (SENT) tests of $[90 / 0]_{s}$ laminates. The transverse direction tension toughness can be obtained from Single Edge Notch Tension (SENT) test. However, in this study it is assumed that this value is the same as the interlaminar mode I toughness because in both cases, toughness is due to matrix failure only. The transverse direction compressive toughness is assumed to be the same as the transverse tension toughness. Shear direction toughness is difficult to measure because it is difficult to obtain a pure shear stable crack propagation. Hence, shear toughness is back calculated from a simulation of the SENT test of a [0] ply laminate. Before the shear test and the corresponding simulation are conducted, items 1-12 should be completed, so that shear toughness is the last unknown variable that needs characterization.

\section{E. Interlaminar failure toughness}

Interlaminar failure toughness and strength are measured using standard test procedures. For mode I toughness and strength, a Double Cantilever Beam (DCB) test is conducted. For mode II toughness, an End Notch Flexure (ENF) test is used. Further, for mode II strength, a Single Lap Joint (SLJ) test is conducted, with a short intact region so that the join failure is strength dominated.

\section{Finite Element Model and results}

EST was integrated into the Abaqus FEM software using the user material Fortran subroutine UMAT for the implicit solver and VUMAT for the explicit solver. ${ }^{26}$ The EST Fortran subroutines are compiled into a static library and linked to Abaqus through the abaqus_v6.env environment file. At each integration point, during each FEM iteration, the UMAT/VUMAT calls the main EST subroutine.

An open hole laminate is modeled using layered shell elements (S4R) with three integration point through the thickness of each lamina. For OHTS simulation, a total displacement of $2 \mathrm{~mm}$ was applied over $1000 \mathrm{sec}$ using the *DYNAMIC, IMPLICIT keyword in Abaqus with the parameter APPLICATION = QUASI-STATIC. For OHCS, a ramped total displacement of $2 \mathrm{~mm}$ was applied using the $*$ EXPLICIT, EXPLICIT keyword in Abaqus. For compression loading, the explicit solver was used to avoid convergence issues cause by a sudden postpeak instability.

Results from the simulations were compared with experiments for four different layups. The nomenclature used to label laminates describe the percentage of $0^{\circ}, 45^{\circ} \& 90^{\circ}$ plies in a laminate. For example laminate $(10 / 70 / 20)$ is made of $10 \% 0^{\circ}$ plies, $70 \% 45^{\circ}$ plies and $20 \% 90^{\circ}$ plies. The maximum load is effect by the stacking sequence.

Results corresponding to the zeroth order, first order, and second order models are shown in figure (13). Although all the predictions are close to experiment, the first and second order models are seen to be a better match with experiments (within 5\%), especially in laminates with high percentage of $0^{\circ}$ plies, as shown in figure 13 . The results are normalized by the compressive strength of the $0^{\circ}$ ply with $\phi=1^{\circ}$ obtained from the Considere construction ie; the zero ply un-notched strength. 
The difference between the zeroth order and second order analysis is more pronounced in tension. Results corresponding to the zeroth order and second order models are shown in figure (14). The results are normalized by the tensile strength of the $0^{\circ}$ ply obtained from an experiment ie; the zero ply un-notched tensile strength. Second order analysis results were found to be within $5 \%$ of the experimental results. First order model for OHTS was not pursued, since the tensile failure mechanisms are better captured by second order model.

\section{Conclusion}

In this paper, different modeling approaches and coupon level test requirements for predicting compressive (OHCS) and tensile (OHTS) strength of laminates with a hole are outlined. A zeroth order approach which utilized Considere construction and Lekhnitski approximations is described. This approach is useful for a efficient approximation of the OHCS and OHTS, as it provides conservative values for OHCS and OHTS.

A first order approach based on global-local micro-mechanical modeling is also outlined. This approach can be used for OHCS prediction and is found to be within $5 \%$ of experimental values. First order approach can capture kink band formation which is the dominant failure mechanism in compression. However, this approach does not capture the damage in off-axis plies within a laminate.

A unified tension compression formulation which captures both damage and failure was finally described. EST formulation addressed both pre-peak and post-peak non-linearities appropriately. Matrix micro-damage, predominantly responsible for the observed pre-peak non-linearity in a composite lamina, was accounted for with a single ISV representing the dissipated potential associated with micro-damage, along the lines of the original Schapery Theory formulation. The relationship between the transverse and shear moduli of the lamina were related to the ISV through a pair of experimentally-obtainable micro-damage functions. Three major in-plane failure mechanisms applicable to continuous fiber-reinforced, laminated, polymer matrix composites were identified: mode I matrix cracks, mode II matrix cracks, and fiber breakage. A failure initiation criterion was used to mark the transition from a damaging continuum to a damaged continuum with an embedded discontinuity. Currently, a quadratic failure criterion is employed, but future work will focus on utilizing a more physics-based initiation criterion. After failure initiation, micro-damage evolution ceases and separate ISVs are introduced to incorporate the effects of the three major failure mechanisms. Evolution of the failure ISVs is based upon traction-separation laws that are material specific and that can be measured. Good agreement is seen with first order predictions of OHCS, and with second order predictions of both OHCS and OHTS. The material used for this study did not show significant delamination. This however is not true for all material systems. Future studies will be aimed at incorporating delamination capability using cohesive zone elements, as reported in. ${ }^{20}$ This "third order" model will be able to capture all possible failure modes associated with PMC laminates, including delamination.

\section{References}

\footnotetext{
${ }^{1}$ A. Waas, C.D. Babcock,jr. and W.G. Knauss, An Experimental Study of Compression Failure of Fibrous Laminates in the Presence of Stress Gradients, International Journal of Solids and Structures, Vol. 26, No. 9/10, pp. 1071-1098, 1990.
} 
${ }^{2}$ C. Soutis, N.A. Fleck and P.A. Smith Failure Prediction Technique for Compression Loaded Carbon Fibre-Epoxy Laminates with Open Holes. Journal of Composite Materials, 25(11): 1476-1498, 1991.

${ }^{3}$ S.W. Yurgartis Measurement of small angle fiber misalignments in continuous fiber composites. Composites Science and Technology, 30: pp279-293, 1987.

${ }^{4}$ J. H. Ahn and A. Waas, A Micromechanics-Based Finite Element Model for Compressive Failure of Notched Uniply Composite Laminates under Remote Biaxial Loads, Trans. ASME J.Eng. Materials and Technology, vol. 121, pp360-366, 1999.

${ }^{5}$ Ahn, J.H. and Waas, AM, Prediction of Compressive Failure in Laminated Composites at Room and Elevated Temperature, AIAA Journal, 40 (2): 346-358, 2002.

${ }^{6} \mathrm{P}$. Davidson and Anthony M. Waas Effect of Fiber Waviness on the Compressive Strength of Unidirectional Carbon Fiber Composites. 53rd AIAA/ASME/ASCE/AHS/ASC Structures, Structural Dynamics and Materials Conference, AIAA 2012-1612, Honolulu, Hawaii, 2012.

${ }^{7}$ W.S Slaughter and N.A. Fleck Compressive Fatigue of Composites. J. Mech. amd Phys. of Solids, 41, No. 8, pp1265-1284, 1993.

${ }^{8}$ S. Lekhnitskii, Anisotropic Elasticity. Mir Publishers, 1980.

${ }^{9}$ W.H. Ng, A.G. Salvi, A.M. Waas, Characterization of the in-situ non-linear shear response of laminated fiber-reinforced composites. Composites Science and Technology, 70, pp1126-1134, 2010.

${ }^{10}$ C. Soutis and P.T. Curtis A Method for Predicting the Fracture Toughness of CFRP, Laminates Failing by Fibre Microbuckling Composites, Part A, 31(7): 733-740, 2000.

${ }^{11}$ C. Soutis and C. Filiou Stress Distributions around Holes in Composite Laminates Subjected to Biaxial Loading Applied Composite Materials, 5: 365378, 1998.

${ }^{12}$ B. Budiansky, N.A. Fleck. Compressive failure of fibre composites. J Mech Phys Solids, 41, pp183-211, 1993.

${ }^{13} \mathrm{C}$. Heinrich and Anthony M. Waas Investigation of progressive damage and fracture in laminated composites using the smeared crack approach 53rd AIAA/ASME/ASCE/AHS/ASC Structures, Structural Dynamics and Materials Conference, AIAA 2012-1537, Honolulu, Hawaii, 2012.

${ }^{14}$ E. Pineda and Anthony M. Waas A Thermodynamically-Based Mesh Objective Work Potential Theory for Predicting Intralaminar Progressive Damage and Failure in Fiber-Reinforced Laminates 53rd AIAA/ASME/ASCE/AHS/ASC Structures, Structural Dynamics and Materials Conference, AIAA 20121612, Honolulu, Hawaii, 2012.

${ }^{15}$ E.J. Pineda and A.M. Waas Modelling progressive failure of fibre reinforced laminated composites: mesh objective calculations Aeronautical Journal, Volume: 116, Issue: 1186, pp1221-1246, 2012.

${ }^{16} \mathrm{P}$. Davidson and Anthony M. Waas. Open hole compressive strength prediction of laminated composite plate, In American Society for Composites 27th Technical Conference, Arlington, TX, 2012, 2012.

${ }^{17}$ S.H. Lee and Anthony M. Waas Compressive Response and Failure of Fiber Reinforced Unidirectional Composites Int J Fracture100 (3), pp275-306, 1999.

${ }^{18}$ S.T. Pinho , P. Robinson, L. Iannucci, Fracture toughness of the tensile and compressive fibre failure modes in laminated composites Composites Science and Technology,66 , 2069-2079, 2006.

${ }^{19}$ J. Llorca, C. Gonzalez, J. Segurado, Finite element and homogenization of materials, in Multiscale Materials Modeling, Fundamentals and Applications, CRC Press, edited by Z.X. Guo, 2007.

${ }^{20}$ W. Ji and A.M. Waas, Progressive Failure Analaysis for the interaction of interlaminar and intralaminar failure modes in composite structures with an initial delamination, Aeronautical Journal, Vol. 117, pp1-14, 2013.

${ }^{21}$ D.L. Sicking, Mechanical Characterization of Nonlinear Laminated Composites with Transverse Crack Growth, Ph.D. thesis, Texas A\&M University, College Station, TX, 1992.

22Z.P. Bažant and B.H. Oh, Crack band theory for fracture of concrete, Mater. and Struct., Vol. 16, pp. $155-77,1993$.

${ }^{23}$ R.A. Schapery, Prediction of compressive strength and kink bands in composites using a work potential, Int. J. Solids Structures, Vol. 32, No. 6, pp739-765, 1995.

${ }^{24}$ S. Basu, Computational Modeling of Progrssive Failure and Damage in Composite Laminates, Ph.D. thesis, University of Michigan, Ann Arbor, MI, 2005.

15 of 26

American Institute of Aeronautics and Astronautics 
${ }^{25}$ S. Basu, A.M. Waas, D.R. Ambur, Compressive failure of fiber composites under multiaxial loading, J. Mech. Phys. Solids, Vol. 54, No. 3, pp. 611-634, 2007.

${ }^{26}$ Abaqus, Abaqus User's Manual, Vol. 1-3, Version 6.10-1, Dassault Systèmes Simulia Corp., Providence, RI, 2008.

16 of 26

American Institute of Aeronautics and Astronautics 


\begin{tabular}{|c|c|c|}
\hline Item & Parameter & Test \\
\hline \multicolumn{3}{|c|}{ Damage: Schapery evolution polynomial } \\
\hline 1 & Shear & {$\left[+45_{2} /-45_{2}\right]_{s}$ tension } \\
\hline 2 & Trans. tension & {$\left[+30_{2} /-30_{2}\right]_{s}$ tension } \\
\hline $3^{\dagger}$ & Trans. compression & {$\left[+30_{2} /-30_{2}\right]_{s}$ compression } \\
\hline \multicolumn{3}{|c|}{ Intralaminar failure strain } \\
\hline 4 & \multirow{5}{*}{$\begin{array}{c}\text { Fiber tension } X_{\epsilon t} \\
\text { Fiber compression } X_{\epsilon c} \\
\text { Trans. tension } Y_{\epsilon t} \\
\text { Trans. compression } Y_{\epsilon c} \\
\text { Shear } Z_{\epsilon}\end{array}$} & [0] tension \\
\hline 5 & & [0] compression or FEM \\
\hline 6 & & [90] tension \\
\hline $7^{\dagger}$ & & $\mathrm{N} / \mathrm{A}$ \\
\hline 8 & & {$\left[+45_{2} /-45_{2}\right]$ tension } \\
\hline \multicolumn{3}{|c|}{ Intralaminar failure toughness } \\
\hline 9 & \multirow{5}{*}{$\begin{array}{l}\text { Fiber tension } G_{I f-t} \\
\text { Fiber compression } G_{I f-c} \\
\text { Trans. tension } G_{I t-t} \\
\text { Trans. compression } G_{I t-c} \\
\quad \text { Shear } G_{I I s}\end{array}$} & CTS $[90 / 0]_{s}$ tension \\
\hline $10^{\dagger}$ & & CCS $[90 / 0]_{s}$ compression \\
\hline $11^{\ddagger}$ & & SENT [90] tension \\
\hline $12^{\dagger}$ & & $\mathrm{N} / \mathrm{A}$ \\
\hline $13^{\ddagger}$ & & SENT $[0]$ tension \& FEM \\
\hline \multicolumn{3}{|c|}{ Interlaminar failure } \\
\hline 14 & Mode I $G_{I}, \sigma_{I}$ & $\mathrm{DCB}$ \\
\hline 15 & Mode II $G_{I I}$ & ENF \\
\hline 16 & Mode II strength $\tau_{c}$ & SLJ \\
\hline \multicolumn{3}{|c|}{$\begin{array}{l}\dagger \text { assumed the same as in tension } \\
\ddagger \text { assumed same as interlaminar failure }\end{array}$} \\
\hline
\end{tabular}

Table 1: Parameters required for modeling 


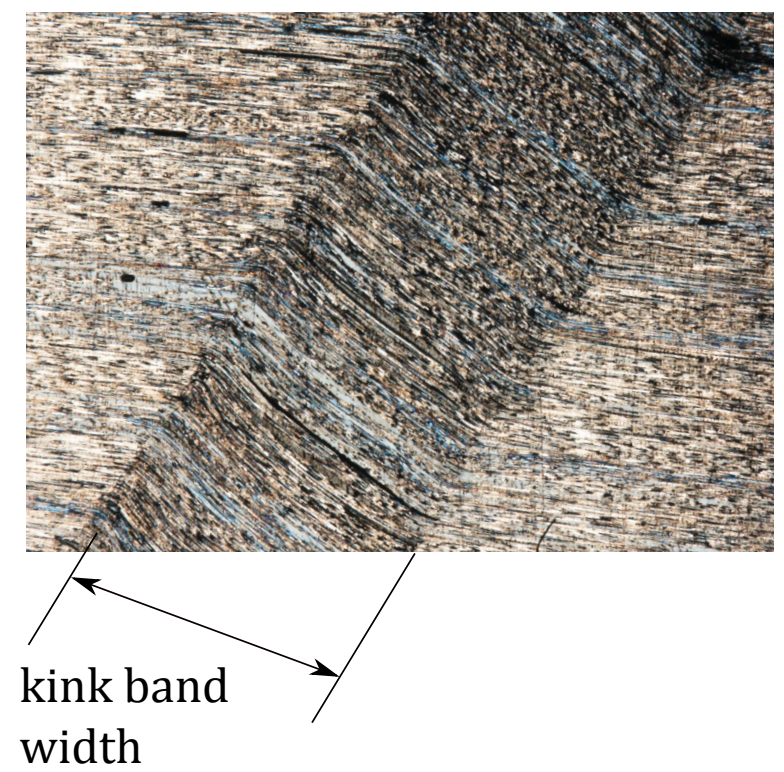

Figure 1: Typical kink band in a carbon fiber composite.

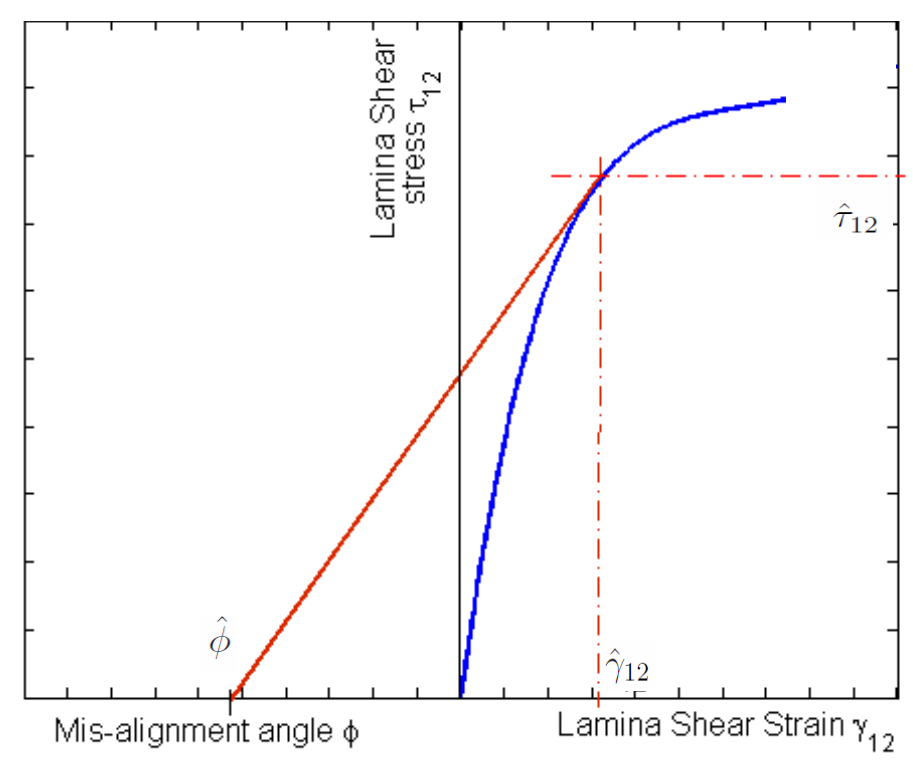

Figure 2: Considere construction

18 of 26

American Institute of Aeronautics and Astronautics 


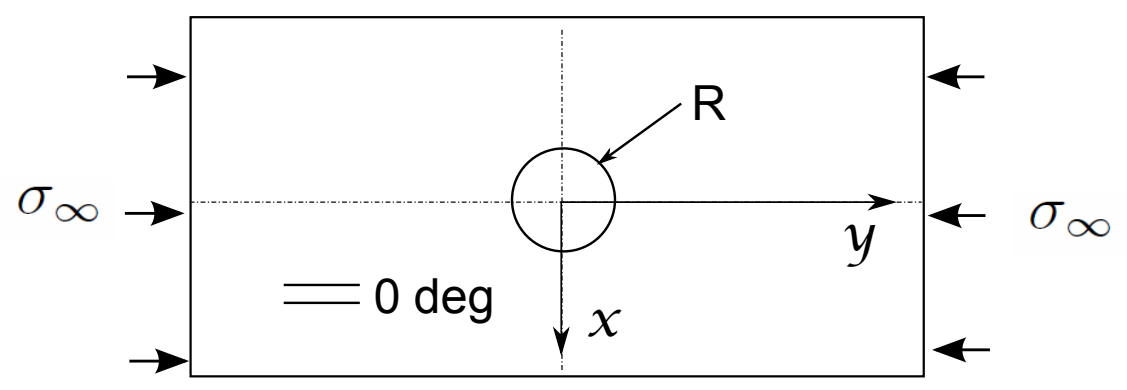

Figure 3: Infinite plate with a hole under compression
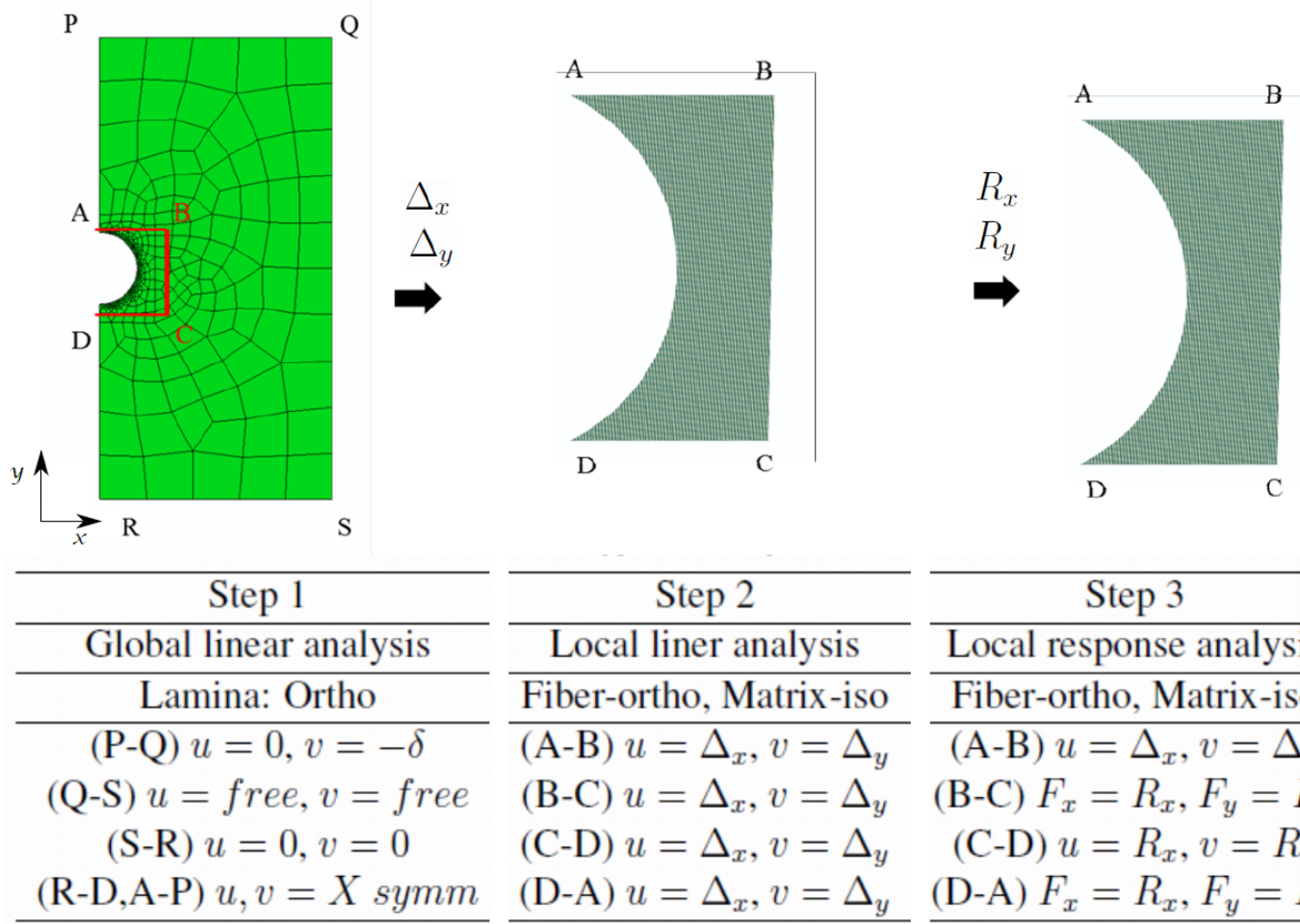

\begin{tabular}{l}
\hline Step 2 \\
\hline Local liner analysis \\
\hline Fiber-ortho, Matrix-iso \\
\hline (A-B) $u=\Delta_{x}, v=\Delta_{y}$ \\
(B-C) $u=\Delta_{x}, v=\Delta_{y}$ \\
(C-D) $u=\Delta_{x}, v=\Delta_{y}$ \\
(D-A) $u=\Delta_{x}, v=\Delta_{y}$
\end{tabular}

\begin{tabular}{c}
\hline Step 3 \\
\hline Local response analysis \\
\hline Fiber-ortho, Matrix-iso \\
\hline (A-B) $u=\Delta_{x}, v=\Delta_{y}$ \\
(B-C) $F_{x}=R_{x}, F_{y}=R_{y}$ \\
(C-D) $u=R_{x}, v=R_{y}$ \\
(D-A) $F_{x}=R_{x}, F_{y}=R_{y}$
\end{tabular}

Figure 4: Steps in global-local analysis 


\section{Pinching in micro-region}

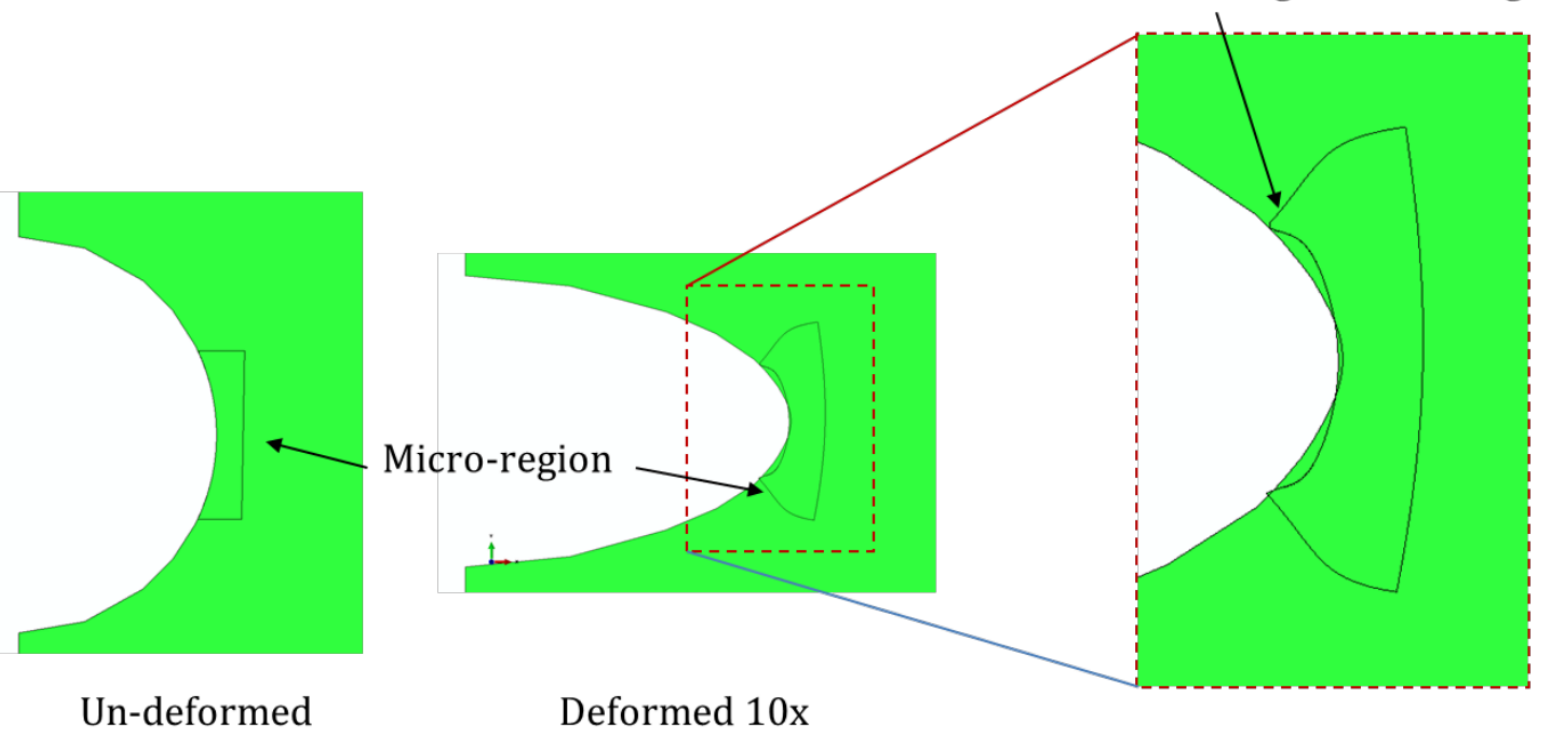

Figure 5: Pinching in local analysis when reaction forces are not applied on the free-edge. 

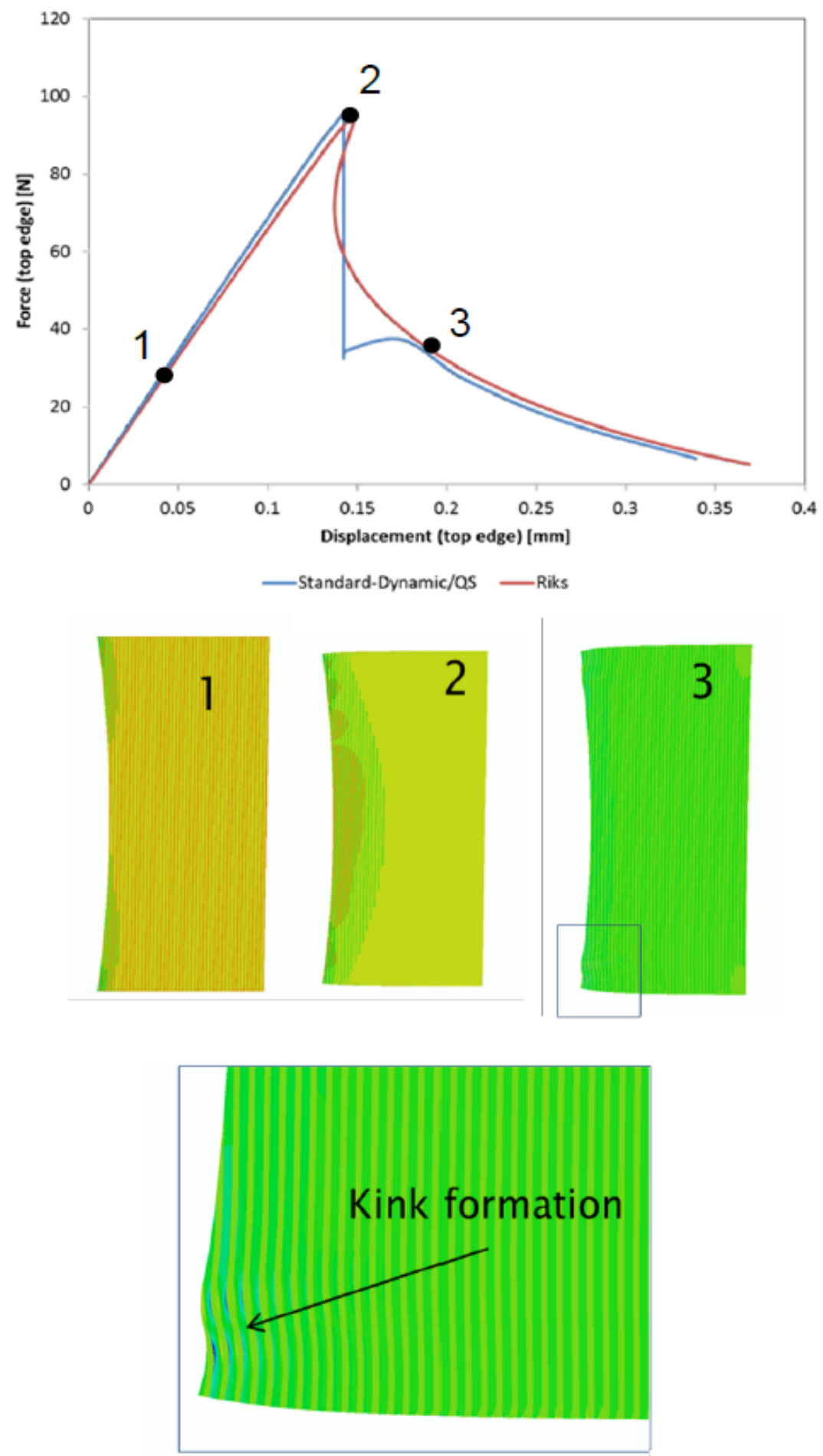

Figure 6: Typical result in global-local analysis. Top: Load displacement plot from response analysis of micro region. Two methods are shown, Standard-dynamic and Riks analysis. Middle: Shows the axial stress plots at load points indicated on the load-displacement curve. Bottom: shows the kink band formation in the micro region. 


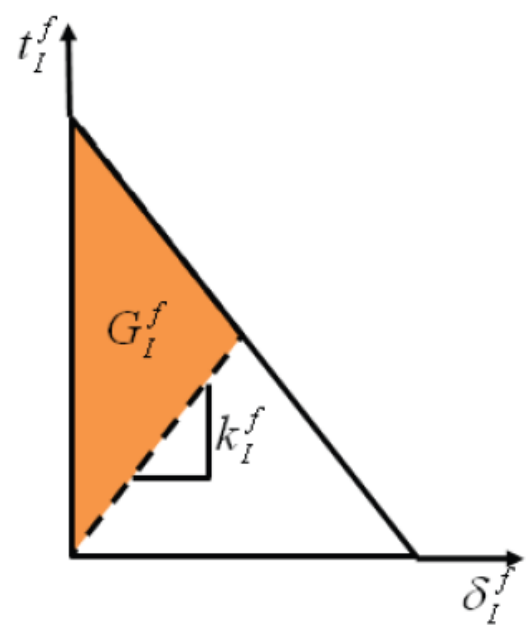

(a) Mode I fiber fracture.

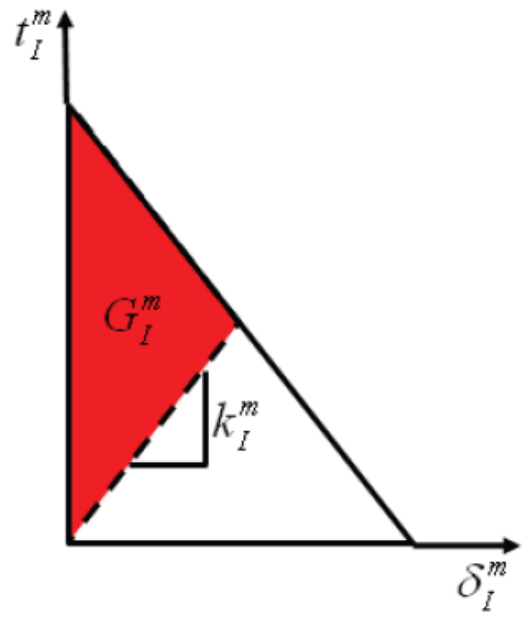

(b) Mode I matrix fracture.

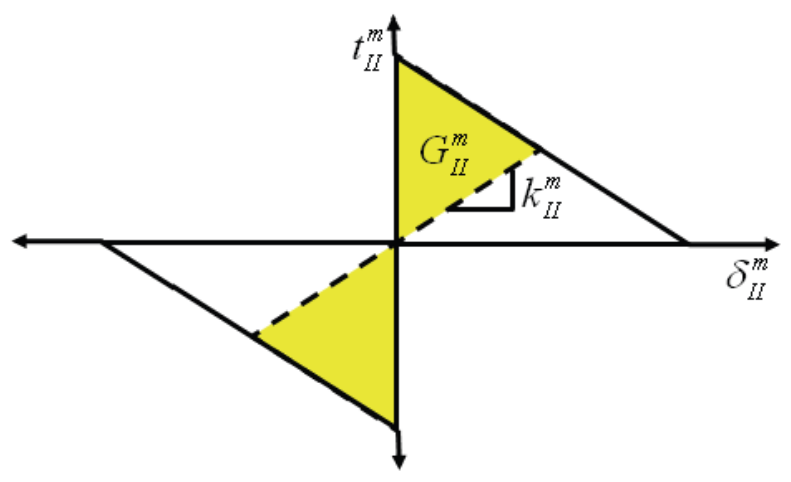

(c) Mode II matrix fracture.

Figure 7: Traction-separation laws used in second order analysis 


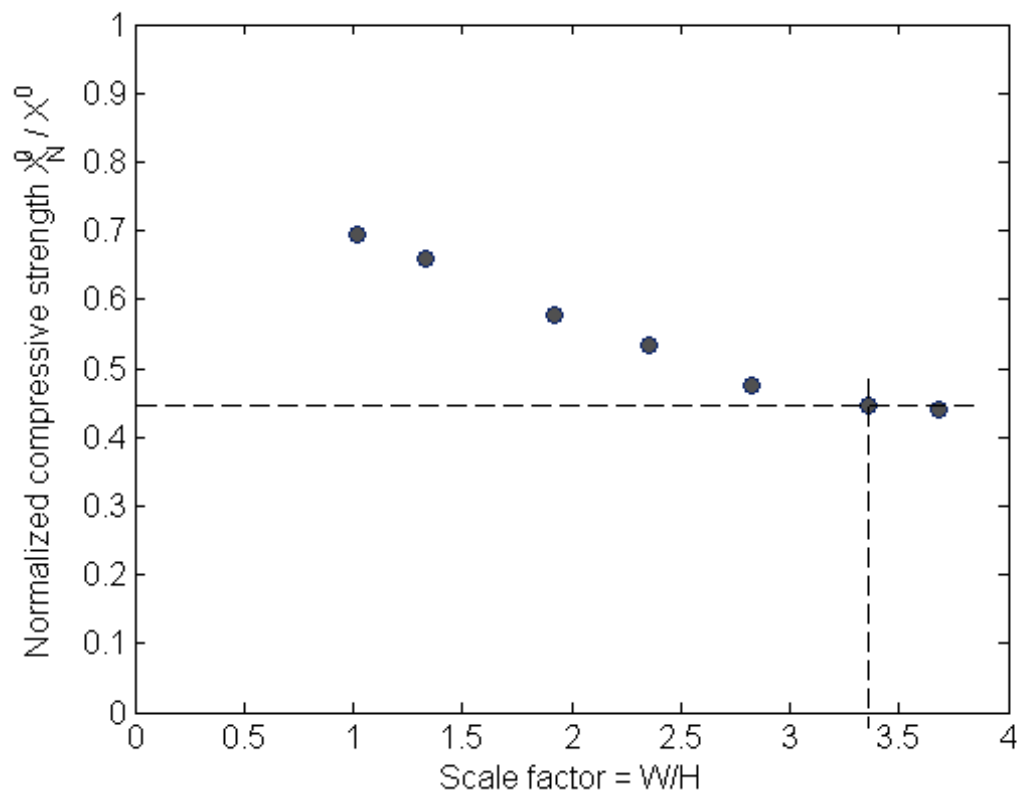

Figure 8: Scaling study result

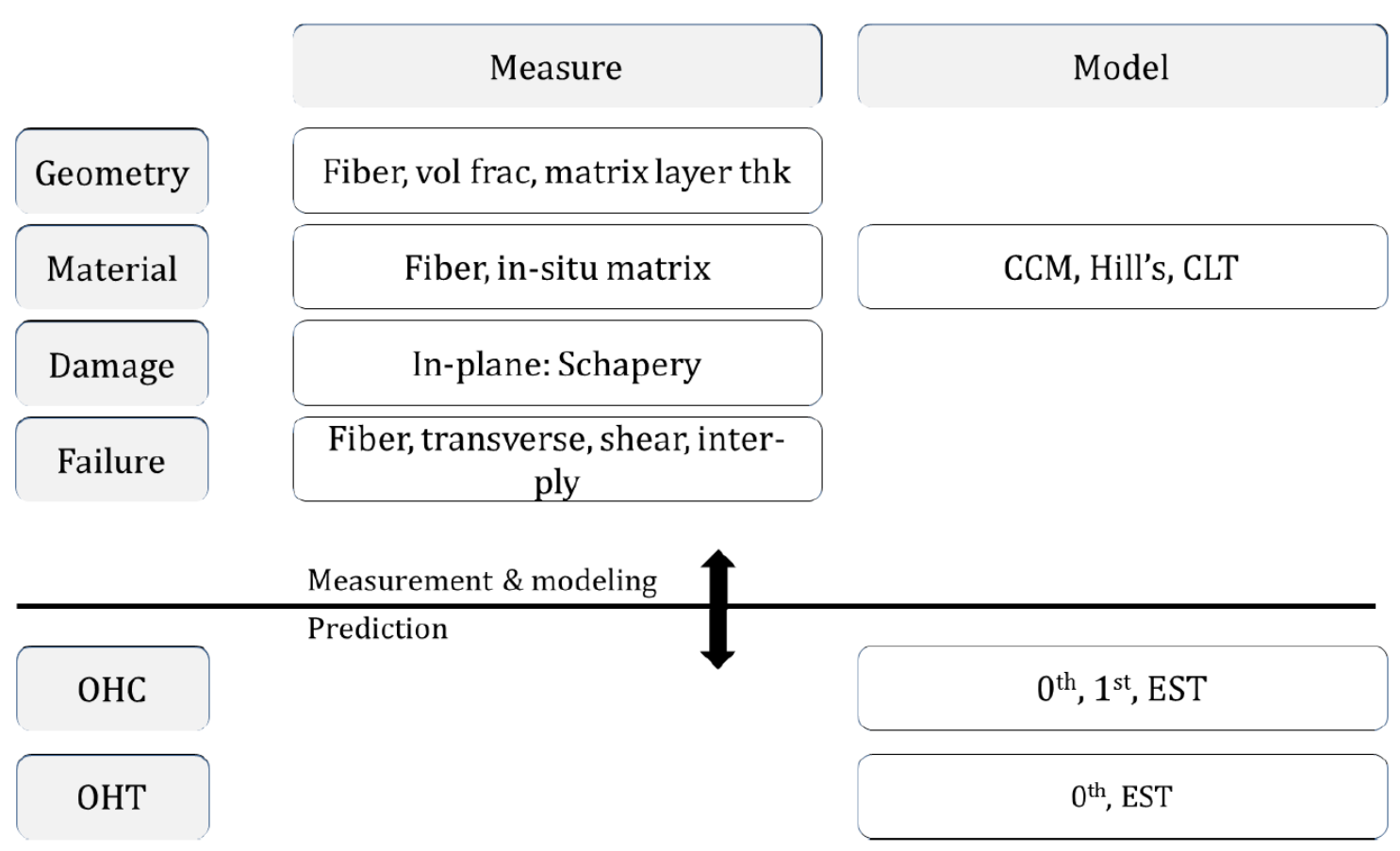

Figure 9: General procedure for OHC \& OHT analysis 


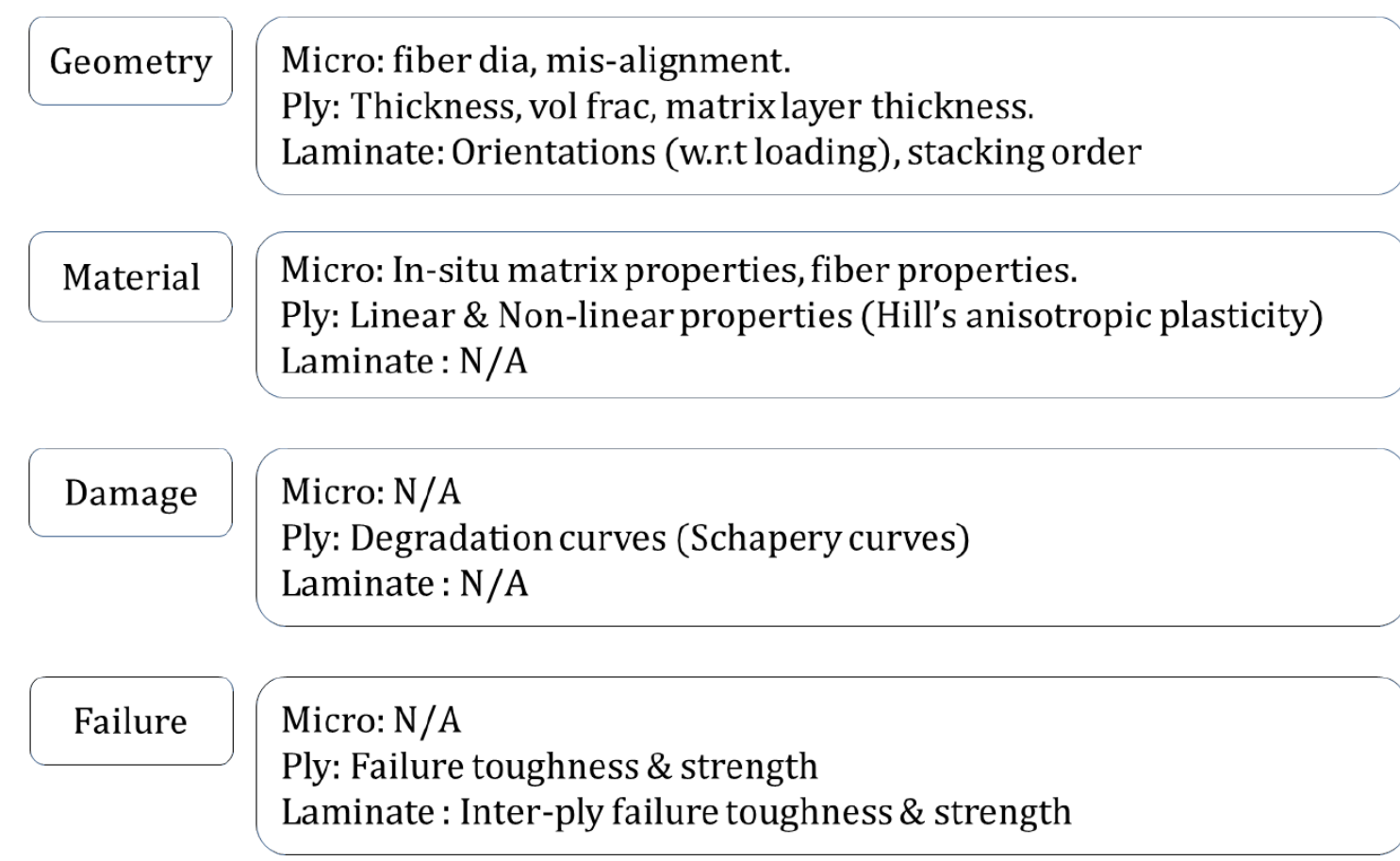

Figure 10: Measurements required for a typical laminate analysis

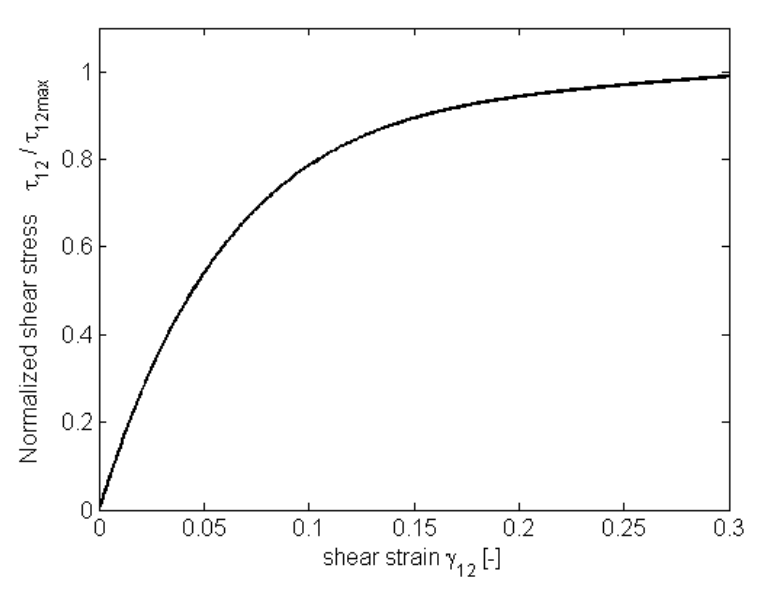

(a) In-situ shear stress-strain

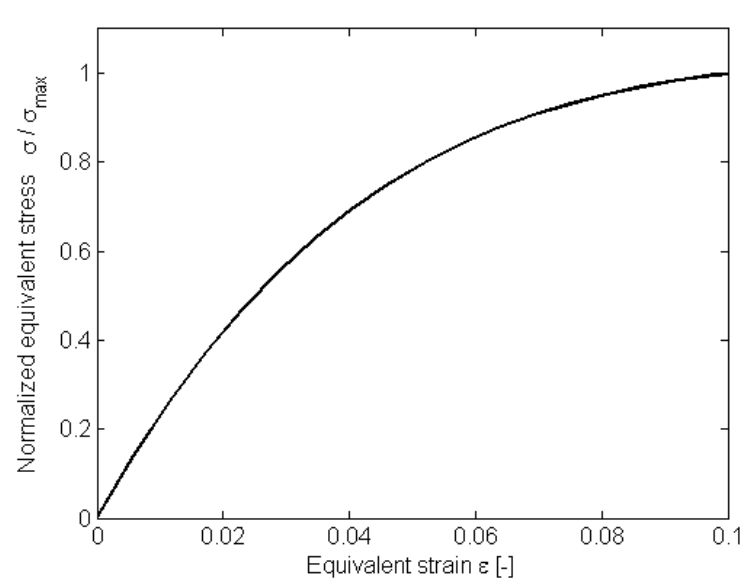

(b) Equivalent stress-strain curve

Figure 11: Matrix properties 


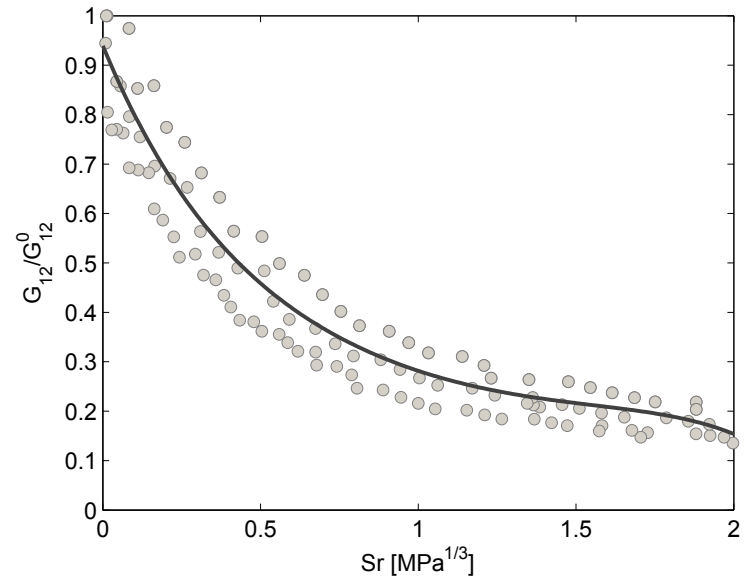

(a) Shear damage curve

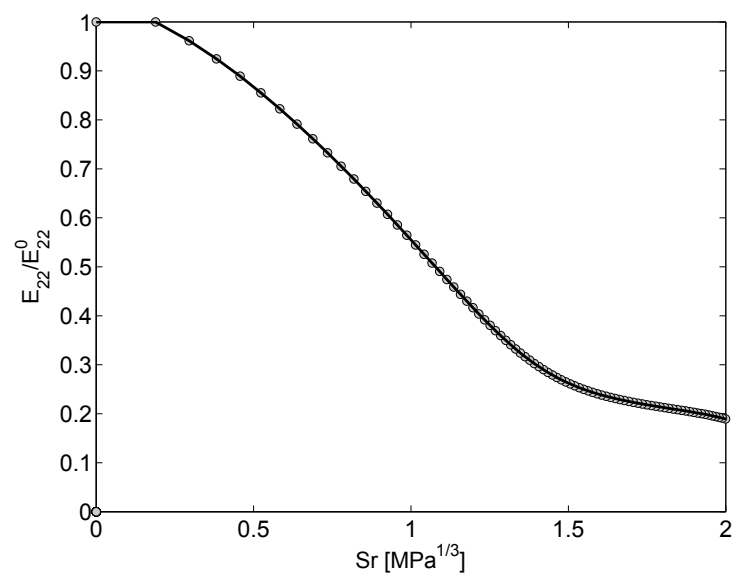

(b) Transverse damage curve

Figure 12: Schapery damage curve

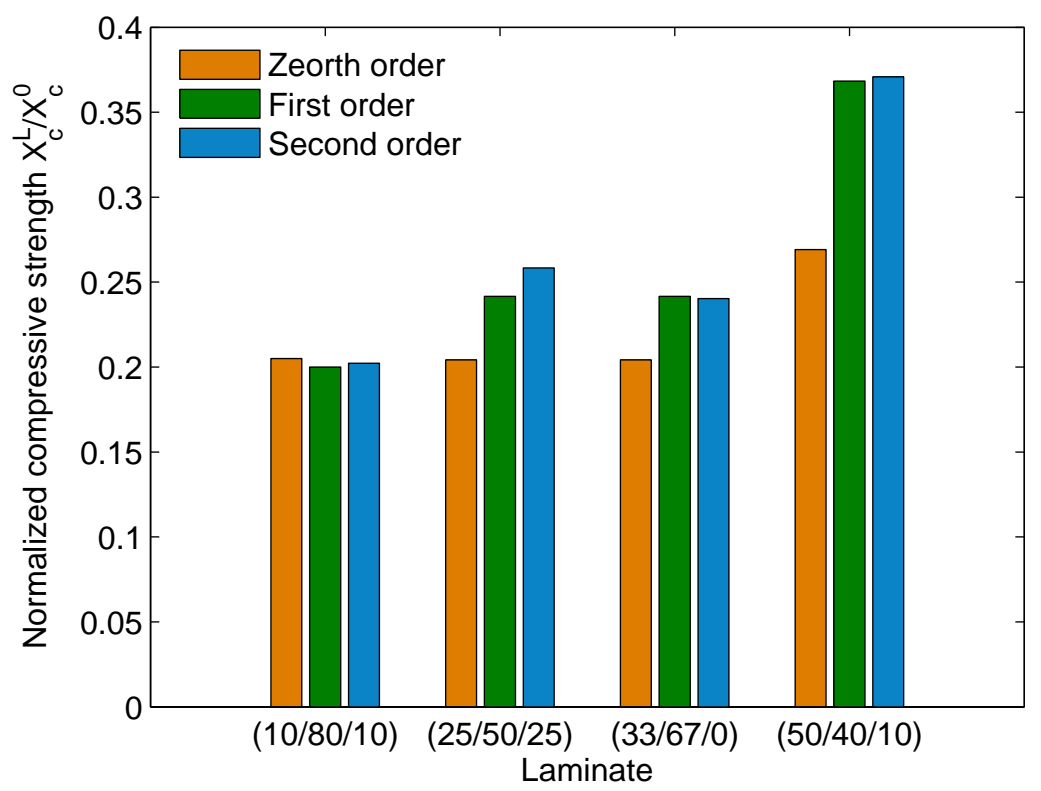

Figure 13: OHCS results 


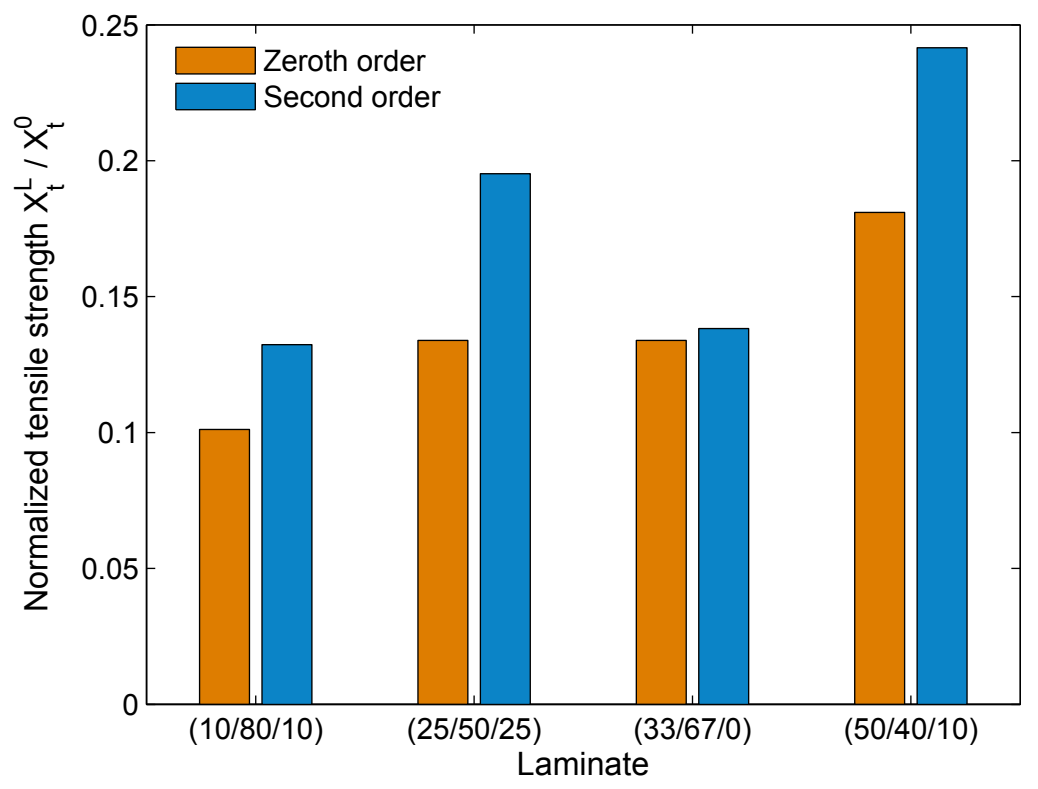

Figure 14: OHTS results 